\title{
Resveratrol reversed chronic restraint stress-induced impaired cognitive function in rats
}

\author{
QIAN ZHANG $^{1}$, XUEER WANG $^{1}$, XUEMEI BAI $^{1}$, YUNKAI XIE $^{1}$, TIANTIAN ZHANG $^{1}$, \\ SHISHI BO ${ }^{1}$ and XIAOYANG CHEN ${ }^{2}$ \\ ${ }^{1}$ Department of Medical Psychology, Shandong University School of Medicine; ${ }^{2}$ Humanities Medical Research Center, \\ Shandong University, Jinan, Shandong 250012, P.R. China
}

Received July 20, 2016; Accepted May 2, 2017

DOI: $10.3892 / \mathrm{mmr} .2017 .6851$

\begin{abstract}
Chronic stress occurs in everyday life, and often results in memory impairments and depressive symptoms. Resveratrol is a natural polyphenol that possesses numerous biological properties, including potent antidepressant-like effects. The present study aimed to examine the effects of resveratrol treatment on chronic restraint stress (CRS)-induced cognitive impairment and to explore the underlying molecular mechanisms. Male Wistar rats were exposed to CRS for 21 days in order to induce depressive-like behavior. The results demonstrated that $\mathrm{CRS}$ (6 h/day, 21 days) was able to induce cognitive deficits in rats, as evidenced by Morris water maze and novel object recognition tests. In addition, CRS exposure significantly decreased the mRNA and protein expression levels of hippocampal brain-derived neurotrophic factor (BDNF) in the rats. Conversely, chronic treatment with resveratrol ( $80 \mathrm{mg} / \mathrm{kg}$, i.p.; 21 days) significantly prevented the behavioral and biochemical alterations induced by CRS. The effects of resveratrol were nearly identical to those observed with fluoxetine treatment. In conclusion, the present study demonstrated that resveratrol may be a potential therapeutic agent for the treatment of chronic stress-induced cognitive impairments, and its underlying molecular mechanism may be associated with the increased levels of hippocampal BDNF.
\end{abstract}

\section{Introduction}

Stress is a well-known environmental factor that can affect human and animals, resulting in behavioral alterations and various diseases (1). In addition, it is considered a physiological

Correspondence to: Dr Xiaoyang Chen, Humanities Medical Research Center, Shandong University, 107 Wenhua Xi Road, Jinan, Shandong 250012, P.R. China

E-mail: 18653199188@163.com

Key words: resveratrol, chronic restraint stress, cognitive deficits, brain-derived neurotrophic factor or psychological response induced by adverse stimuli, which may disturb the functioning of an organism (2). It has previously been reported that cognitive impairments are common in stress-associated mental disorders, and chronic stress was able to impair learning and memory (3). Furthermore, exposure to chronic stress results in atrophy and functional impairment in several key brain regions, including the frontal cortex and hippocampus (4). The hippocampus, which is a limbic region important in learning and memory, is particularly susceptible to chronic stress $(5,6)$.

Chronic restraint stress (CRS) is a specific and classic method that can be used to simultaneously induce psychological and physiological stress. Previous studies have demonstrated that CRS is able to impair cognitive hippocampus-dependent functions, particularly learning and memory $(7,8)$. Brain-derived neurotrophic factor (BDNF) serves an important role in hippocampal learning and memory, and is affected by CRS; this has been hypothesized to be one of the main mechanisms underlying the impaired cognitive function $(9,10)$. Although previous studies have focused on this mechanism $(9,10)$, at present, few studies have investigated the effectiveness of therapeutic agents for the treatment of chronic stress-induced cognitive deficits.

Resveratrol is a natural polyphenolic compound present in grapes and red wine, which exhibits numerous beneficial properties, including anticancer, antioxidant, anti-aging, anti-inflammatory and antiallergenic activities (11). Previous studies have demonstrated that resveratrol exerts powerful neuroprotective effects $(12,13)$. Notably, it has been demonstrated that resveratrol protects against cognitive impairment in animal models of Alzheimer's disease, depression or vascular dementia (14-16). The underlying protective mechanisms of resveratrol are associated with the upregulation of cAMP response element-binding protein-BDNF or the reduction of oxidative stress. A recent study revealed that chronic resveratrol administration exhibited beneficial effects on spatial memory retention following global cerebral ischemia (17). However, the protective effects of resveratrol on CRS-induced cognitive deficits and the underlying molecular mechanisms remain unclear. Therefore, the present study aimed to explore the neuroprotective effects of resveratrol on CRS-induced cognitive dysfunction and its molecular mechanisms. 


\section{Materials and methods}

Animals. A total of 50 adult male Wistar rats (age, 8 weeks; weight, $180-200 \mathrm{~g}$ ) were purchased from Laboratory Animal Center, Shandong University (Jinan, China). The rats were maintained under standard laboratory conditions (temperature, $20 \pm 2^{\circ} \mathrm{C} ; 12$-h light/dark cycle) and had free access to food and water. Animals were acclimated to laboratory conditions for 1 week prior to experimentation. The experimental protocol was approved by the Ethics Committee of Shandong University.

Drug administration and experimental groups. Resveratrol was purchased from Sigma-Aldrich (Merck KGaA, Darmstadt, Germany), and was dissolved in absolute ethanol before being diluted to the desired concentration. Resveratrol was administered intraperitoneally at a dose of $80 \mathrm{mg} / \mathrm{kg}$ to rats daily for 21 days. Fluoxetine was purchased from Lilly S.A. (Madrid, Spain). Fluoxetine (10 mg/kg), freshly suspended in saline, was administered intragastrically daily for 21 days.

The rats were divided into five groups: The control, resveratrol, CRS, CRS + resveratrol, and CRS + fluoxetine groups. All drugs were administered $30 \mathrm{~min}$ prior to exposure to CRS for 21 days.

CRS procedure. The restrainers were comprised of transparent plastic tubes (height, $5 \mathrm{~cm}$; width, $5.5 \mathrm{~cm}$; length, $22 \mathrm{~cm}$ ). The rats were subjected to $6 \mathrm{~h}$ of restraint stress daily for 21 days, during which they were deprived of food and water, without physical suppression.

Open field test. On day 22, an open field test was conducted. Locomotor activity was measured in an open field, which consisted of a 90x90 cm gray wooden floor divided into 36 equal squares by red lines, surrounded by $45 \mathrm{~cm}$ high boundary walls. Each rat was placed in the center of the floor and behavioral parameters, including crossing, rearing and grooming, were counted for $5 \mathrm{~min}$.

Morris water maze (MWM) test. On days 23-28 a MWM test was conducted. Spatial learning and memory performance was determined using the MWM test, as previously described with minor modifications (18). The MWM consisted of a black cylindrical tank (diameter, $120 \mathrm{~cm}$ ) with a circular escape platform (diameter, $10 \mathrm{~cm}$ ). The tank was filled with water $\left(21-24^{\circ} \mathrm{C}\right)$ that was dyed with the addition of an atoxic acrylic black dye. The maze was divided into four equal quadrants and the platform was submerged $2 \mathrm{~cm}$ beneath the surface of the water. A tracking system was used to measure the performance of the rats (SMART polyvalent video-tracking system; Panlab, Barcelona, Spain). Each rat was subjected to 5 consecutive acquisition trials to find the hidden platform. The rats were allowed $60 \mathrm{sec}$ to locate the hidden platform. The time taken to escape onto the hidden platform (escape latency) was recorded by the tracking software. A total of $24 \mathrm{~h}$ following the navigation test, the probe test was performed to assess reference memory, during which the platform was withdrawn. The activity of the rats was monitored for $60 \mathrm{sec}$. The latency to enter the target quadrant and the total time spent in the target quadrant were recorded.

Novel object recognition task (NORT). On days 29-31 the NORT experiment was conducted. The basic design of the NORT was similar to that described by Torner et al (19). The apparatus was a Plexiglas cage $(60 \times 40 \times 40 \mathrm{~cm})$ with an exchangeable floor. A total of 2 days prior to testing the rats were allowed to explore for $15 \mathrm{~min}$. The objects to be discriminated were water-filled plastic bottles and their weight was such that the rats could not displace them. The objects and the apparatus were cleaned to remove the olfactory cues after each trial.

Each session consisted of two trials: Acquisition phase and retrieval phase. In the acquisition phase, two different objects were placed in the box and the animals were allowed to explore for $10 \mathrm{~min}$. The inter-trial interval was $60 \mathrm{~min}$. During the retrieval phase, one object from the acquisition phase was replaced with a duplicate object, and the rats were allowed to explore for a further $10 \mathrm{~min}$. The objects and their positions were counterbalanced within each session.

The measure was the time spent by rats exploring the objects. A ratio reflecting the discrimination between the novel and the familiar object was calculated, as follows: Novel/(novel + familiar time).

Reverse transcription-quantitative polymerase chain reaction $(R T-q P C R)$. Following the NORT, rats were sacrificed and their hippocampi were dissected. Total RNA was isolated from the rat hippocampus using TRIzol reagent (Invitrogen; Thermo Fisher Scientific, Inc., Waltham, MA, USA). Total RNA $(2 \mu \mathrm{g})$ was then reverse-transcribed into cDNA using a commercial RT-PCR kit (Fermentas; Thermo Fisher Scientific, Inc.) according to manufacturer's protocol. A total of $20 \mu \mathrm{l}$ cDNA was subjected to qPCR amplification using the UltraSYBR mixture (Cowin Bioscience Co., Ltd., Beijing, China) according to the manufacturer's protocol. The thermocycling protocol was as follows: Initial denaturation at $95^{\circ} \mathrm{C}$ for $10 \mathrm{~min}$, followed by 40 cycles at $95^{\circ} \mathrm{C}$ for $10 \mathrm{sec}$, and at $58^{\circ} \mathrm{C}$ for $30 \mathrm{sec}$. At the end of the PCR reaction, a melting curve was obtained by holding the temperature at $95^{\circ} \mathrm{C}$ for $15 \mathrm{sec}$, cooling to $60^{\circ} \mathrm{C}$ for $1 \mathrm{~min}$, and heating slowly at a rate of $0.5^{\circ} \mathrm{C} / \mathrm{s}$ up to $95^{\circ} \mathrm{C}$. The primer sequences were as follows: BDNF forward, 5'-AGC TGA GCG TGT GTG ACA GT-3' and reverse, 5'-ACC CAT GGG ATT ACA CTT GG-3'; and $\beta$-actin forward, 5'-TGG AAT CCT GTG GCA TCC ATG AAA C-3' and reverse, 5'-TAA AAC GCA GCT CAG TAA CAG TCC G-3'. Relative expression levels of BDNF were analyzed using the $2^{-\Delta \Delta C q}$ method and normalized to the expression of GAPDH (20).

Determination of BDNF expression. BDNF protein expression was measured in hippocampal tissue using an ELISA kit (cat no. EK0309; Wuhan Boster Biological Technology, Ltd., Wuhan, China), according to the manufacturer's protocol. The samples and protein standards were added to a 96-well plate. After incubation and washing, the detection antibody was added to the wells, followed by the addition of a horseradish peroxidase conjugate. After incubation and washing, the stop solution was added. The optical density was measured at 

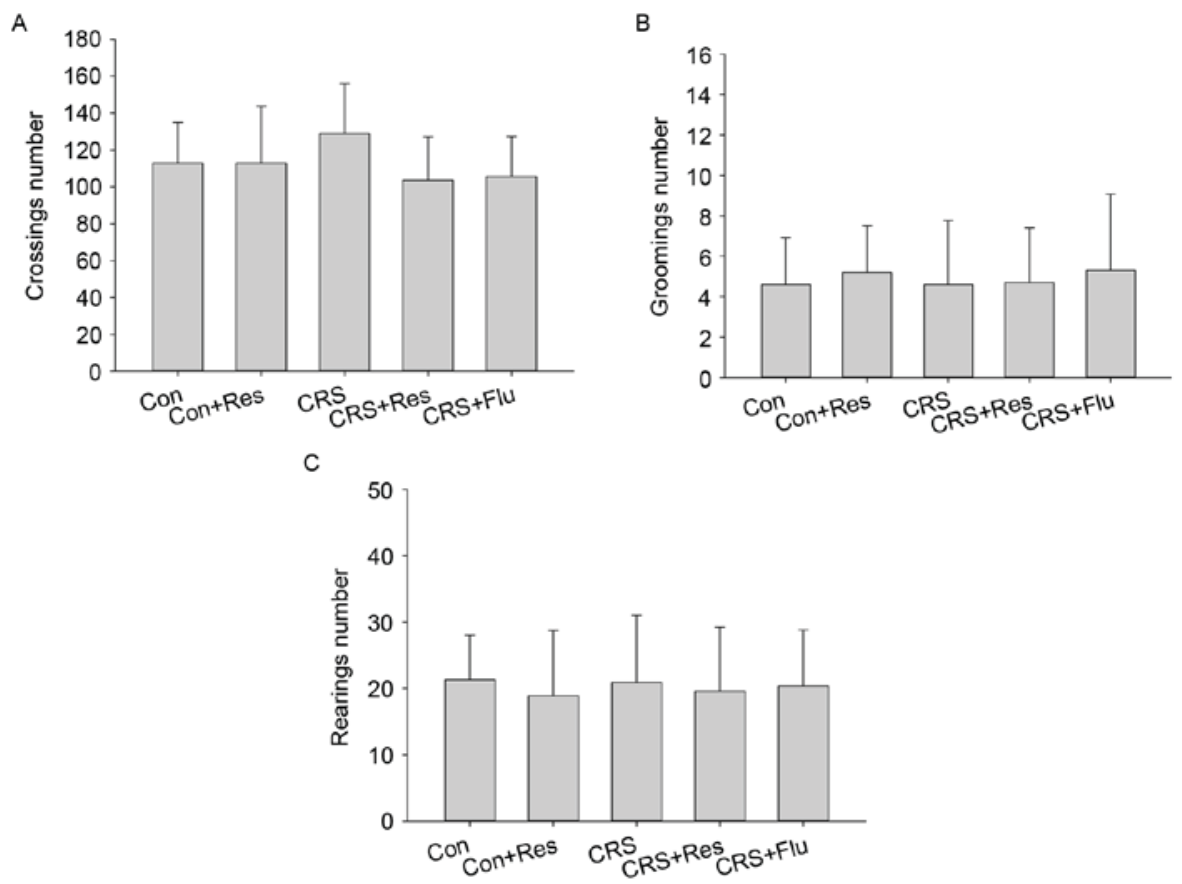

Figure 1. Effects of CRS and resveratrol on behavioral alterations in an open field test. Number of (A) crossings, and (B) grooming and (C) rearing behaviors. Data are presented as the mean \pm standard deviation ( $\mathrm{n}=10$ rats/group). Con, control; CRS, chronic restraint stress; Res, resveratrol; Flu, fluoxetine.

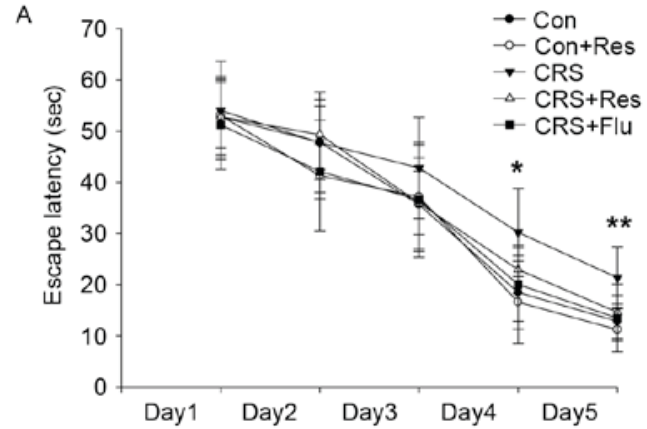

B

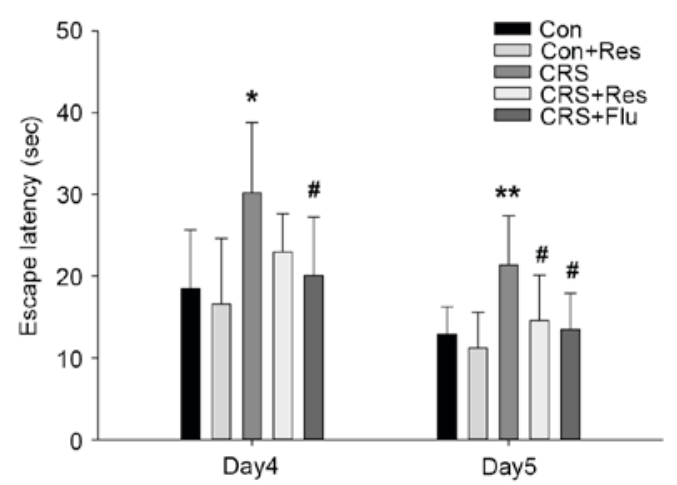

Figure 2. Effects of CRS and resveratrol during the acquisition trials in the Morris water maze test. (A) Escape latency in the acquisition trial. (B) Escape latency on days 4 and 5 . Data are presented as the mean \pm standard deviation ( $\mathrm{n}=10$ rats/group). ${ }^{*} \mathrm{P}<0.05,{ }^{* * *} \mathrm{P}<0.01$ vs. Con group; $\mathrm{P}<0.05$ vs. $\mathrm{CRS}$ group. Con, control; CRS, chronic restraint stress; Res, resveratrol; Flu, fluoxetine.

$450 \mathrm{~nm}$ using a microplate reader (Bio-Rad Laboratories, Inc., Hercules, CA, USA).

Statistical analysis. Statistical analysis was performed using SPSS software version 19.0 (IBM Corp., Armonk, NY, USA).
Data are presented as the mean \pm standard deviation of 4- 6 independent experiments. Data from the MWM test were analyzed with repeated measures analysis of covariance (ANCOVA) followed by a post hoc Tukey-Kramer test. The other data were analyzed by one-way analysis of variance followed by a post hoc Tukey honest significant difference test. $\mathrm{P}<0.05$ was considered to indicate a statistically significant difference.

\section{Results}

Effects of CRS and resveratrol on locomotor activity in the open field test. As presented in Fig. 1, analysis of the results obtained in the open field test yielded no statistically significant differences in the number of crossings $[\mathrm{F}(4,49)=1.535$, $\mathrm{P}>0.05]$, and grooming $[\mathrm{F}(4,49)=0.138, \mathrm{P}>0.05]$ and rearing behaviors $[F(4,49)=0.116, P>0.05]$ between the groups.

Effects of CRS and resveratrol on learning and memory in MWM test. As presented in Fig. 2A, with regards to learning, a repeated measures ANCOVA of escape latency revealed a significant improvement during the 5 days of training $[\mathrm{F}(4,49)$ $=210.343, \mathrm{P}<0.001]$, however there was no association between treatments and days $[F(4,49=0.998, P>0.05]$, suggesting that animals in each group behaved similarly regardless of the previous treatments. In addition, the stressed rats reached the platform significantly later than the control rats on days 4 $(\mathrm{P}<0.05)$ and $5(\mathrm{P}<0.01)$. Treatment with resveratrol and fluoxetine significantly decreased the latency compared with the CRS group on day 5 ( $\mathrm{P}<0.05$, Fig. $2 \mathrm{~B})$.

As shown in Fig. 3, rats exposed to CRS spent more time reaching the platform compared with the control group $(\mathrm{P}<0.05)$; however, treatment with resveratrol or fluoxetine significantly decreased the latency to reach the platform compared with the rats in the CRS group $(\mathrm{P}<0.05)$. 


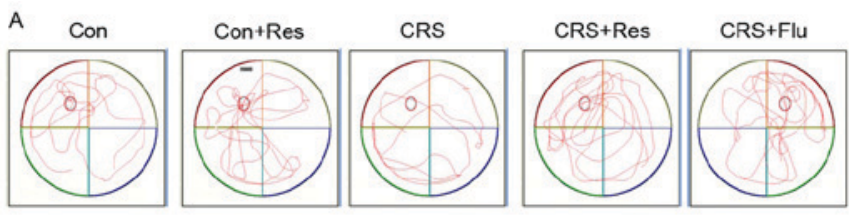

B

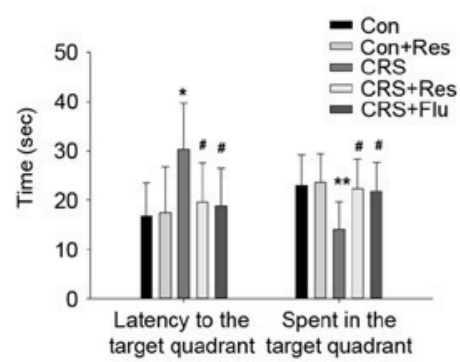

Figure 3. Effects of CRS and resveratrol during the probe trial in the Morris water maze test. (A) Diagram indicating the movement of the rats on day 6 . (B) Escape latency and time spent in the target quadrant. Data are presented as the mean \pm standard deviation ( $\mathrm{n}=10$ rats/group). ${ }^{*} \mathrm{P}<0.05,{ }^{* *} \mathrm{P}<0.01$ vs. Con group; ${ }^{\text {P }}<0.05$ vs. CRS group. Con, control; CRS, chronic restraint stress; Res, resveratrol; Flu, fluoxetine.

Furthermore, compared with the control group, rats in the CRS group spent less time in the target quadrant $(\mathrm{P}<0.01)$, whereas administration of resveratrol or fluoxetine significantly increased the time spent in the target quadrant compared with the CRS group $(\mathrm{P}<0.05$, Fig. 3B).

Effects of CRS and resveratrol administration on NORT. As presented in Fig. 4A, there were no significant differences in the exploration time between the groups during the acquisition phase $[\mathrm{F}(4,49)=0.306, \mathrm{P}>0.05]$. However, the results of an ANOVA revealed significant alterations in the exploration time between the groups in the retrieval phase $[\mathrm{F}(4,49)=5.563$, $\mathrm{P}<0.01]$. Post hoc analysis demonstrated that rats exposed to CRS exhibited a shorter exploration time compared with the control group $(\mathrm{P}<0.01)$. Furthermore, rats treated with resveratrol or fluoxetine spent longer exploring the novel object during the retrieval phase compared with the CRS group $(\mathrm{P}<0.05$, Fig. 4B). As shown in Fig. 4C, rats in the CRS group exhibited a decreased discrimination ratio compared with the control group $(\mathrm{P}<0.01)$, whereas treatment with resveratrol or fluoxetine significantly increased the discrimination ratio $(\mathrm{P}<0.01)$.

Effects of CRS and resveratrol on BDNF expression. As presented in Fig. 5, there were significant alterations in the BDNF mRNA $[\mathrm{F}(4,49)=15.299, \mathrm{P}<0.001]$ and protein $[F(4,49)=6.234, P<0.001]$ expression levels between the groups in the hippocampus. CRS significantly decreased BDNF mRNA $(\mathrm{P}<0.001$, Fig. 5A) and protein levels in the hippocampus $(\mathrm{P}<0.01$, Fig. $5 \mathrm{~B})$ compared with the control rats. In addition, administration of resveratrol or fluoxetine markedly reversed CRS-induced reductions in BDNF mRNA and protein expression (Fig. 5).

\section{Discussion}

The present study demonstrated that treatment with resveratrol prevented CRS-induced cognitive impairment in rats. In
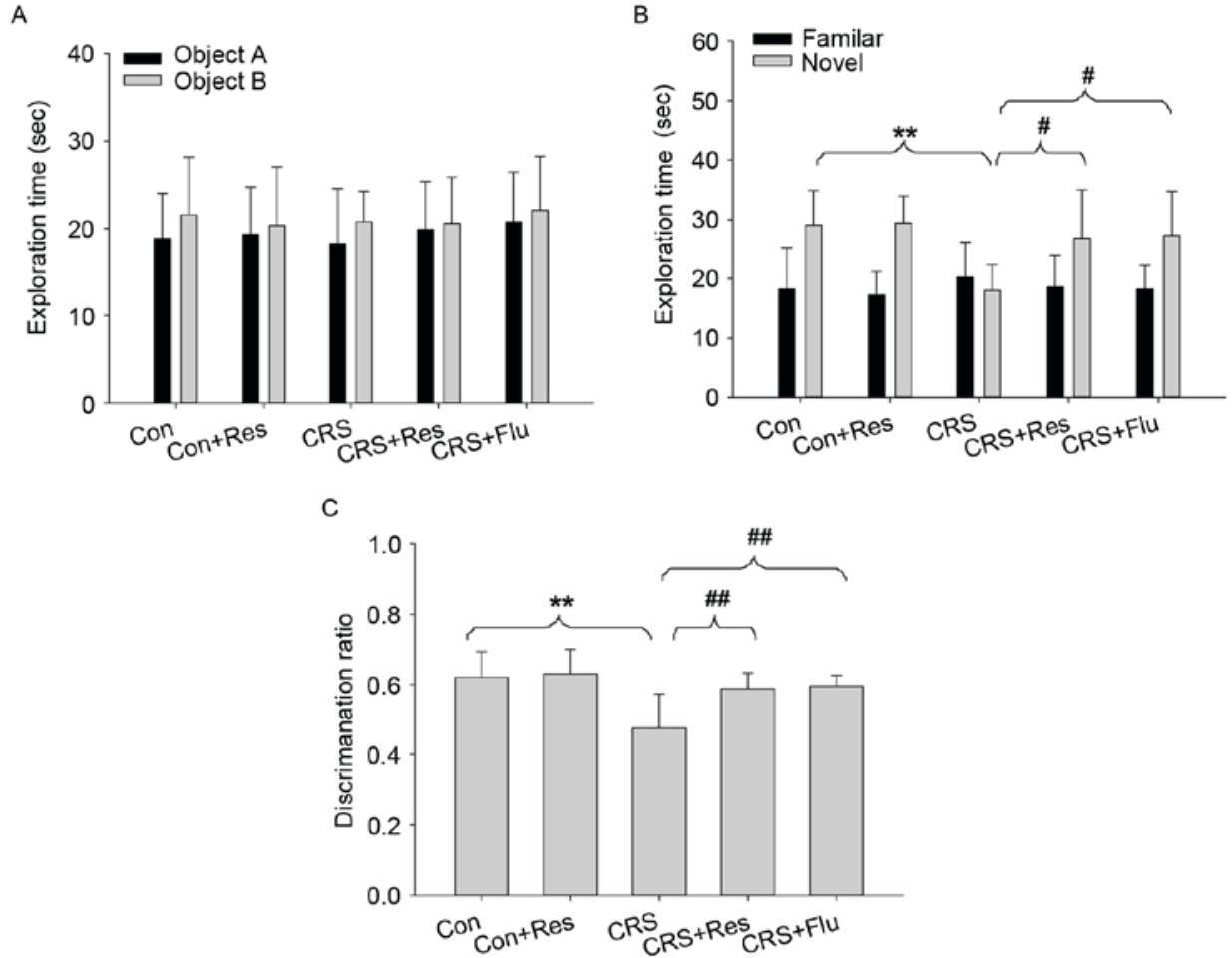

Figure 4. Effects of CRS and resveratrol on recognition memory performance in the novel object recognition task. (A) Results of the acquisition phase, columns represent the mean time exploring objects A and B. (B) Results of the retrieval phase, columns represent the mean time exploring novel and familiar objects. (C) Discrimination ratio was calculated. Data are presented as the mean \pm standard deviation ( $\mathrm{n}=10$ rats/group). ${ }^{* *} \mathrm{P}<0.01 \mathrm{vs}$. Con group; ${ }^{\#} \mathrm{P}<0.05$, ${ }^{\# \#} \mathrm{P}<0.01$ vs. CRS group. Con, control; CRS, chronic restraint stress; Res, resveratrol; Flu, fluoxetine. 
A

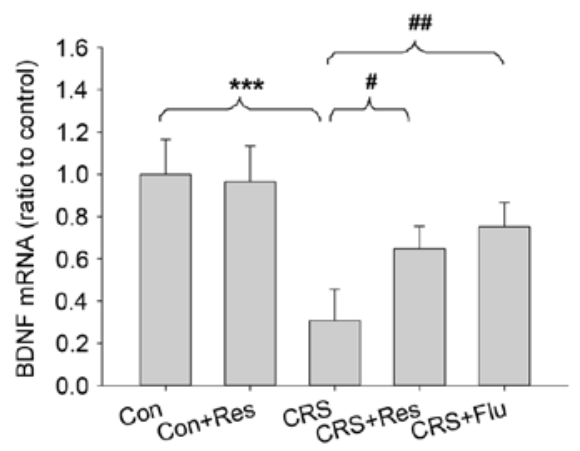

B

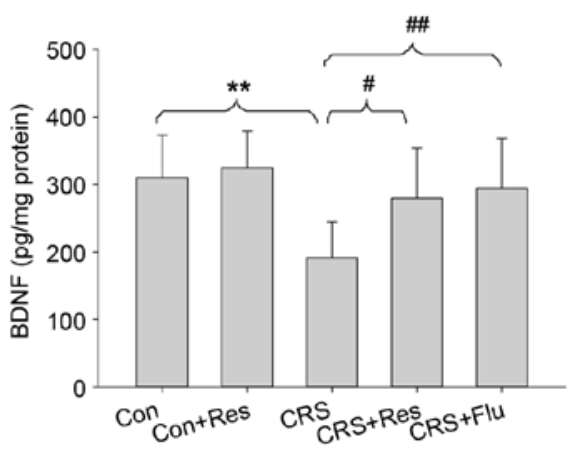

Figure 5. Effects of CRS and resveratrol on BDNF expression in the hippocampus. (A) mRNA expression levels of BDNF were determined by reverse transcription-quantitative polymerase chain reaction. The graph presented includes results obtained from 4 independent experiments. (B) Protein expression levels of BDNF were determined by ELISA. Data are presented as the mean \pm standard deviation $(\mathrm{n}=10 \mathrm{rats} / \mathrm{group})$. ${ }^{* * *} \mathrm{P}<0.01,{ }^{* * * *} \mathrm{P}<0.001 \mathrm{vs}$. Con group; ${ }^{\#} \mathrm{P}<0.05,{ }^{\# \#} \mathrm{P}<0.01$ vs. CRS group. Con, control; CRS, chronic restraint stress; Res, resveratrol; Flu, fluoxetine; BDNF, brain-derived neurotrophic factor

addition, resveratrol increased the mRNA and protein expression levels of BDNF in the hippocampus of CRS-exposed rats. These results were similar to those observed in rats treated with fluoxetine.

To investigate whether resveratrol can alleviate CRS-induced impaired cognitive function, MWM test and NORT were used to explore learning and memory ability. In the MWM test, rats treated with resveratrol took less time to reach the platform compared with the rats in the CRS group. In addition, treatment with resveratrol increased the time spent in the target quadrant compared with the rats in the CRS group. These data indicated that resveratrol was able to prevent CRS-induced learning and memory impairment in rats. In the open field test, the results revealed that there was no statistical difference among the experimental groups, indicating that CRS and drug administration had no effect on locomotor activity, which would not interfere with performance in the MWM test. Similar results were also obtained in the NORT and these data were comparable to those in the rats treated with fluoxetine. These findings suggested that resveratrol may exhibit a protective role in alleviating CRS-induced cognitive impairment.

The hippocampus is one of the most important regions in the brain, and is a primary structure for learning and memory (21). BDNF, which is an important member of the neurotrophin family of growth factors, is widely distributed in the hippocampus and cerebral cortex (22). BDNF helps to support the survival of existing neurons, encourages the growth and differentiation of neurons and synapses (23), and promotes the formation of long-term memory (24). Previous studies have demonstrated that CRS decreases the expression of BDNF in the hippocampus and induces impaired cognitive function $(25,26)$. Recently, resveratrol has been reported to increase the expression of BDNF in various in vivo and in vitro models (27-29). In addition, it has been indicated that resveratrol is able to reverse stress-induced impaired cognitive function $(15,30)$. Consistent with these findings, the results of the present study demonstrated that resveratrol was able to prevent CRS-induced cognitive deficit in rats, as evidenced by the MWM test and NORT. Furthermore, the CRS-induced decrease in BDNF mRNA and protein expression was reversed by treatment with resveratrol. These results suggested that the molecular mechanism underlying the protective effects of resveratrol may be associated with upregulation of hippocampal BDNF expression in rats.

In conclusion, the present study demonstrated that resveratrol may serve a crucial role in preventing CRS-induced cognitive deficits in rats. It may be hypothesized that these neuroprotective effects may be due to resveratrol-mediated alterations in BDNF expression in the hippocampus. These results indicated the possibility of using resveratrol as a therapeutic agent to prevent stress-induced cognitive impairment.

\section{Acknowledgements}

The present study was supported by funding from the National Natural Science Foundation of China (grant no. 81200879) and the Fundamental Research Funds of Shandong University (grant no. 2015JC008).

\section{References}

1. Dayi A, Cetin F, Sisman AR, Aksu I, Tas A, Gönenc S and Uysal N: The effects of oxytocin on cognitive defect caused by chronic restraint stress applied to adolescent rats and on hippocampal VEGF and BDNF levels. Med Sci Monit 21: 69-75, 2015.

2. Johnson EO, Kamilaris TC, Chrousos GP and Gold PW: Mechanisms of stress: A dynamic overview of hormonal and behavioral homeostasis. Neurosci Biobehav Rev 16: 115-130, 1992.

3. Mohammadi HS, Goudarzi I, Lashkarbolouki T, Abrari K and Elahdadi Salmani M: Chronic administration of quercetin prevent spatial learning and memory deficits provoked by chronic stress in rats. Behav Brain Res 270: 196-205, 2014.

4. Wang Y, Kan H, Yin Y, Wu W, Hu W, Wang M, Li W and Li W: Protective effects of ginsenoside Rg1 on chronic restraint stress induced learning and memory impairments in male mice. Pharmacol Biochem Behav 120: 73-81, 2014.

5. Nagata K, Nakashima-Kamimura N, Mikami T, Ohsawa I and Ohta S: Consumption of molecular hydrogen prevents the stress-induced impairments in hippocampus-dependent learning tasks during chronic physical restraint in mice. Neuropsychopharmacology 34: 501-508, 2009.

6. Aggleton JP, Vann SD, Oswald CJ and Good M: Identifying cortical inputs to the rat hippocampus that subserve allocentric spatial processes: A simple problem with a complex answer. Hippocampus 10: 466-474, 2000. 
7. Ghadrdoost B, Vafaei AA, Rashidy-Pour A, Hajisoltani R, Bandegi AR, Motamedi F, Haghighi S, Sameni HR and Pahlvan S: Protective effects of saffron extract and its active constituent crocin against oxidative stress and spatial learning and memory deficits induced by chronic stress in rats. Eur J Pharmacol 667: 222-229, 2011.

8. Huang RR, Hu W, Yin YY, Wang YC, Li WP and Li WZ: Chronic restraint stress promotes learning and memory impairment due to enhanced neuronal endoplasmic reticulum stress in the frontal cortex and hippocampus in male mice. Int $\mathrm{J}$ Mol Med 35: 553-559, 2015.

9. Ortiz JB, Mathewson CM, Hoffman AN, Hanavan PD, Terwilliger EF and Conrad CD: Hippocampal brain-derived neurotrophic factor mediates recovery from chronic stress-induced spatial reference memory deficits. Eur J Neurosci 40: 3351-3362, 2014.

10. Radahmadi M, Alaei H, Sharifi MR and Hosseini N: Effects of different timing of stress on corticosterone, BDNF and memory in male rats. Physiol Behav 139: 459-467, 2015.

11. Gambini J, Inglés M, Olaso G, Lopez-Grueso R, Bonet-Costa V, Gimeno-Mallench L, Mas-Bargues C, Abdelaziz KM, Gomez-Cabrera MC, Vina J and Borras C: Properties of resveratrol: In vitro and in vivo studies about metabolism, bioavailability and biological effects in animal models and humans. Oxid Med Cell Longev 2015: 837042, 2015.

12. Tsai SK, Hung LM, Fu YT, Cheng H, Nien MW, Liu HY, Zhang FB and Huang SS: Resveratrol neuroprotective effects during focal cerebral ischemia injury via nitric oxide mechanism in rats. J Vasc Surg 46: 346-353, 2007.

13. Kiziltepe U, Turan NN, Han U, Ulus AT and Akar F: Resveratrol, a red wine polyphenol, protects spinal cord from ischemia-reperfusion injury. J Vasc Surg 40: 138-145, 2004.

14. Kumar A, Naidu PS, Seghal N and Padi SS: Neuroprotective effects of resveratrol against intracerebroventricular colchicine-induced cognitive impairment and oxidative stress in rats. Pharmacology 79: 17-26, 2007.

15. Liu D, Zhang Q, Gu J, Wang X, Xie K, Xian X, Wang J, Jiang $\mathrm{H}$ and Wang $\mathrm{Z}$ : Resveratrol prevents impaired cognition induced by chronic unpredictable mild stress in rats. Prog Neuropsychopharmacol Biol Psychiatry 49: 21-29, 2014.

16. Ma X, Sun Z, Liu Y, Jia Y, Zhang B and Zhang J: Resveratrol improves cognition and reduces oxidative stress in rats with vascular dementia. Neural Regen Res 8: 2050-2059, 2013.

17. Girbovan C, Kent P, Merali Z and Plamondon H: Dose-related effects of chronic resveratrol administration on neurogenesis, angiogenesis, and corticosterone secretion are associated with improved spatial memory retention following global cerebral ischemia. Nutr Neurosci 19: 352-368, 2016.
18. Morris R: Developments of a water-maze procedure for studying spatial learning in the rat. J Neurosci Methods 11: 47-60, 1984.

19. Torner L, Tinajero E, Lajud N, Quintanar-Stéphano A and Olvera-Cortés E: Hyperprolactinemia impairs object recognition without altering spatial learning in male rats. Behav Brain Res 252: 32-39, 2013.

20. Livak KJ and Schmittgen TD: Analysis of relative gene expression data using real-time quantitative PCR and the 2(-Delta Delta C(T)) method. Methods 25: 402-408, 2001.

21. Estrada NM and Isokawa M: Metabolic demand stimulates CREB signaling in the limbic cortex: Implication for the induction of hippocampal synaptic plasticity by intrinsic stimulus for survival. Front Syst Neurosci 3: 5, 2009.

22. Yamada $K$ and Nabeshima T: Brain-derived neurotrophic factor/TrkB signaling in memory processes. J Pharmacol Sci 91: 267-270, 2003.

23. Huang EJ and Reichardt LF: Neurotrophins: Roles in neuronal development and function. Annu Rev Neurosci 24: 677-736, 2001.

24. Bekinschtein P, Cammarota M, Katche C, Slipczuk L, Rossato JI, Goldin A, Izquierdo I and Medina JH: BDNF is essential to promote persistence of long-term memory storage. Proc Natl Acad Sci USA 105: 2711-2716, 2008.

25. Kwon DH, Kim BS, Chang H, Kim YI, Jo SA and Leem YH: Exercise ameliorates cognition impairment due to restraint stress-induced oxidative insult and reduced BDNF level. Biochem Biophys Res Commun 434: 245-251, 2013.

26. Yan W, Zhang T, Jia W, Sun X and Liu X: Chronic stress impairs learning and hippocampal cell proliferation in senescence-accelerated prone mice. Neurosci Lett 490: 85-89, 2011.

27. Ali SH, Madhana RM, K V A, Kasala ER, Bodduluru LN, Pitta S, Mahareddy JR and Lahkar M: Resveratrol ameliorates depressive-like behavior in repeated corticosterone-induced depression in mice. Steroids 101: 37-42, 2015.

28. Song J, Cheon SY, Jung W, Lee WT and Lee JE: Resveratrol induces the expression of interleukin-10 and brain-derived neurotrophic factor in BV2 microglia under hypoxia. Int J Mol Sci 15: 15512-15529, 2014.

29. Hurley LL, Akinfiresoye L, Kalejaiye O and Tizabi Y: Antidepressant effects of resveratrol in an animal model of depression. Behav Brain Res 268: 1-7, 2014.

30. Yazir Y, Utkan T, Gacar N and Aricioglu F: Resveratrol exerts anti-inflammatory and neuroprotective effects to prevent memory deficits in rats exposed to chronic unpredictable mild stress. Physiol Behav 138: 297-304, 2015. 